\title{
Simulating the WiMAX Physical Layer in Rayleigh Fading Channel
}

\author{
Jamal Mountassir ${ }^{*}$, Horia Balta, Marius Oltean, Maria Kovaci, Alexandru Isar \\ Department of Communications, University "Politehnica”, Timisoara, 300223, Romania
}

\begin{abstract}
WiMAX is a wireless technology which offers high data rate transmission in broadband. In this paper, the architecture of a WiMAX physical layer simulator is presented. The main blocks are implemented with the aid of the Matlab programming language and the bit error rate (BER) curves in Rayleigh fading channel, are presented and commented.
\end{abstract}

Keywords WiMAX, OFDM, Rayleigh Channel, Simulator

\section{Introduction}

Worldwide Interoperability for Microwave Access, known as WiMAX, is a wireless networking technology which aims for addressing interoperability across IEEE 802.16 standard-based products; it includes the definition of the medium access control (MAC) and the physical (PHY) layer. The IEEE $802.16 \mathrm{e}$ is designed to provide system for mobile broadband access.

The MAC layer is an interface between PHY layer and network architecture. It has a connection-oriented architecture that is designed to support a variety of applications, including voice and multimedia services. The system offers support for constant bit rate, variable bit rate, real-time, and non-real-time traffic flows, in addition to best-effort data traffic. WiMAX MAC is designed to support a large number of users, with multiple connections per terminal, each with its own quality of service (QoS) requirement.

The multiple accesses of users are realized by adjacent and distributed sub-carriers permutations which are defined to formulate sub-carriers to sub-channel mapping for various diversity options: Full Usage of Sub-channels (FUSC), Tile Usage of Sub-carriers (TUSC), Partial Usage of Sub-channels (PUSC) and Adaptive Modulation and Coding (AMC). For the separation of the Down Link (DL) and Up Link (UL) transmissions, the standards IEEE 802.16-2004 and IEEE 802.16e-2005 support both time division duplexing (TDD) and frequency division duplexing (FDD), as well as a half-duplex FDD, which allows for a low-cost system implementation. TDD is preferred in the majority of implementations because of its advantages such as flexibility in choosing uplink-to-downlink data rate ratios and the ability

* Corresponding author:

mountassir.jamal@gmail.com (Jamal Mountassir)

Published online at http://journal.sapub.org/jwnc

Copyright (C) 2011 Scientific \& Academic Publishing. All Rights Reserved to exploit channel reciprocity. For connections that require enhanced reliability, WiMAX supports automatic retransmission requests (ARQ) at the link layer level. ARQ-enabled connections require each transmitted packet to be acknowledged by the receiver; unacknowledged packets are assumed to be lost and are retransmitted. WiMAX also optionally supports hybrid-ARQ, which is an effective hybrid between forward error correction (FEC) and ARQ.

The basics of the PHY layer in WIMAX are presented in Section II. The proposed simulator is described in Section III. Some simulation results are presented and commented in Section IV. The last Section is dedicated to conclusions and further work objectives.

\section{WiMAX Physical Layer}

The WiMAX physical layer is based on the orthogonal frequency division multiplexing (OFDM), which is the transmission scheme of choice to enable the high-speed data, video, and multimedia communications. OFDM is an elegant and efficient scheme for high data rate transmission in non-line-of-sight (NLOS) or multi-path radio environment.

The transmitted base-band signal, which is an ensemble of the signals in all the sub-carriers, can be represented as:

$$
X(t)=\sum_{l=0}^{l-1} S[l] . e^{-j 2 \pi\left(\Delta f+l B B_{c}\right)}, 0 \leq t \leq T
$$

where $S[l]$ is the symbol carried on the $l^{\text {th }}$ sub-carrier; $B c$ is the frequency separation between two adjacent sub-carriers, also referred to as the sub-carrier bandwidth; $\Delta f$ is the frequency of the first sub-carrier; and $T$ is the total useful symbol duration (without the cyclic prefix). At the receiver, the symbol sent on a specific sub-carrier is retrieved by integrating the received signal with a complex conjugate of the tone signal over the entire symbol duration $T$. If the time and the frequency synchronization between the receiver and the transmitter are perfect, the orthogonality between the sub-carriers is preserved at the receiver. When the time and/or frequency synchronization between the transmitter 
and the receiver is not perfect, the orthogonality between the sub-carriers is lost, resulting in inter-carriers interference (ICI). Timing mismatch can occur due to misalignment of the clocks at the transmitter and the receiver and propagation delay of the channel.

Frequency mismatch can occur owing to relative drift between the oscillators at the transmitter and the receiver and nonlinear channel effects, such as Doppler shift. The flexibility of the WiMAX PHY layer allows one to make an optimum choice of various PHY layer parameters, such as cyclic prefix length, number of sub-carriers, sub-carrier separation, and preamble interval, such that the performance degradation owing to inter-symbol interference (ISI) is minimal without compromising the performance. The concept of independently modulating multiple orthogonal frequency tones with narrowband symbol streams is equivalent to first constructing the entire OFDM signal in the frequency domain and then using an inverse fast Fourier transform (IFFT) to convert the signal into the time domain. The IFFT method is easier to implement, as it does not require multiple oscillators to transmit and receive the OFDM signal. In the frequency domain, each OFDM symbol is created by mapping the sequence of symbols on the sub-carriers.

The available sub-carriers may be divided into several groups of sub-carriers called sub-channels. Sub-channels may be constituted using either contiguous sub-carriers or sub-carriers pseudo randomly distributed across the frequency spectrum. Sub-channels formed using distributed sub-carriers provide more frequency diversity, which is particularly useful for mobile applications. WiMAX defines several sub-canalization schemes based on distributed carriers for both the uplink and the downlink.

Several permutation types are defined by the standard, suited for different purposes. FUSC makes full usage of the achievable frequency diversity: any available sub-carrier within the whole spectrum may be allocated to any sub-channel. PUSC firstly splits the available sub-carriers in several groups (segments). The sub-carriers are then mapped to sub-channels within every segment, independently. This approach is still oriented to frequency diversity, and allows a kind of "virtual sectorization": different regions of the cell will correspond to different segments. The PUSC sub-canalization is mandatory for all mobile. By coordinating planning, the neighbor cells may use the same frequency band, the segmentation allowing reducing the interference.

AMC is the name given to an adjacent permutation: the sub-carriers that compose the sub-channels are adjacent in the spectrum. This approach reduces the complexity and is well suited for channels that change slowly in time. Channel estimation is easier due to the fact that carriers are adjacent and consequently the link adaptation becomes simpler too, which explains the name given to this permutation.

Although frequency diversity is lost, band AMC allows system designers to exploit multi-user diversity, allocating sub-channels to users based on their frequency response. Multi-user diversity can provide significant gains in overall system capacity, if the system strives to provide each user with a sub-channel that maximizes its received signal to interference and noise ratio (SINR). In general, contiguous sub-channels are more suited for fixed and low-mobility applications. The WiMAX PHY layer is also responsible for slot allocation and framing over the air. The minimum time-frequency resource that can be allocated by a WiMAX system to a given link is called a slot.

Each slot consists of one sub-channel over one, two, or three OFDM symbols, depending on the particular sub-canalization scheme used. Fig. 1 shows an OFDMA and OFDM frame when operating in TDD mode.

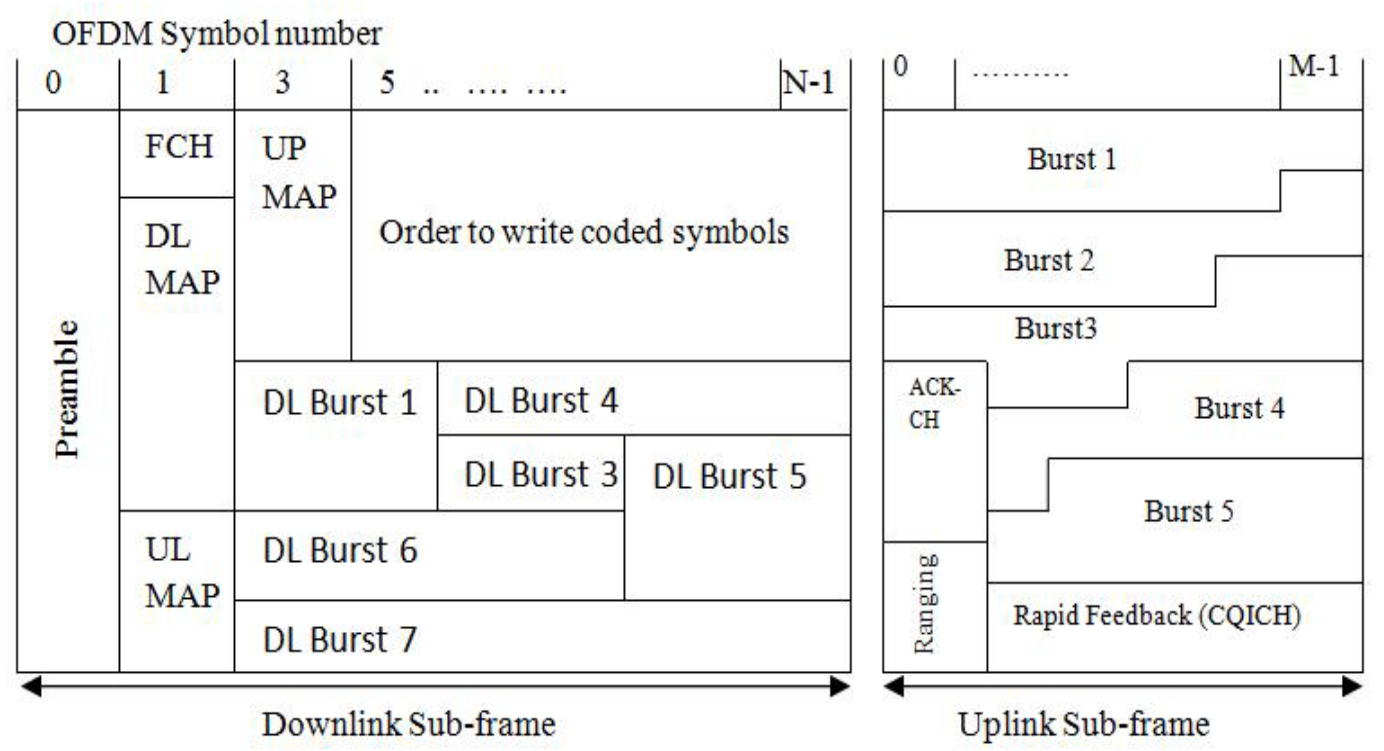

Figure 1. A sample TDD frame structure for Mobile WiMAX 
The frame is divided into two sub-frames: a downlink frame followed by an uplink frame after a small guard interval. The downlink sub-frame begins with a downlink preamble that is used for physical-layer procedures, such as time and frequency synchronization and initial channel estimation. The downlink preamble is followed by a frame control header $(\mathrm{FCH})$, which provides frame configuration information, such as the MAC management messages (MAP) as message length, the modulation, and coding scheme, and the usable sub-carriers. Multiple users are allocated data regions within the frame, and these allocations are specified in the uplink and downlink MAP messages (DL-MAP and UL-MAP) that are broadcasted following the FCH in the downlink sub-frame. MAP messages include the burst profile for each user, which defines the modulation and coding scheme used in that link. Since MAP contains critical information that needs to reach all users, it is often sent over a very reliable link, such as BPSK with rate $1 / 2$ coding and repetition coding. Although the MAP messages are an elegant way for the base station (BS) to inform the various users of its allocations and burst profiles on a per-frame basis, it could form a significant overhead, particularly when there are a large number of users with small packets (e.g., VoIP) for which allocations need to be specified. To mitigate the overhead concern, mobile WiMAX systems can optionally use multiple sub-MAP messages where the dedicated control messages to different users are transmitted at higher rates, based on their individual SINR conditions. The broadcast MAP messages may also optionally be compressed for additional efficiency. An example of physical layer logical units defined in WiMAX for DL-PUSC is shown in figure. 2. An important step of the PHY layer processing is the channel coding. This is linked to the concept of AMC too. Thus, users with good radio conditions may be scheduled to transmit with a high-level modulation (e.g. 64 QAM) and with a high rate of coding.

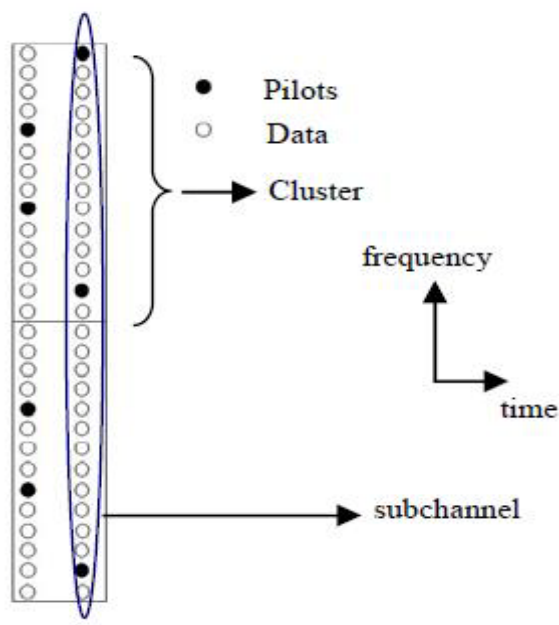

Figure 2. Logical resources for DL-PUS

As channel coding techniques, several options are proposed by the standard: convolutional coding, turbo-coding and LDPC coding. However, most of the manufacturers prefer convolutional Turbo codes (CTC) that achieve a good trade-off between complexity and performance. The digital modulation schemes that are proposed for WiMAX are QPSK, 16QAM and 64QAM. In combination with different coding rates, these modulations compose the modulation and coding profiles, used in the AMC process (e.g. QPSK $1 / 2,16$ QAM $1 \frac{1}{2}$ etc.).

\section{WiMAX Physical Layer Simulator}

The PHY layer simulator block scheme is shown in fig. 3. Its structure is based on[4]. Its blocks implement the PHY layer processing operations described in the previous section. The simulator eases the understanding of the PHY layer signal processing steps. Furthermore, it allows assessing the BER performance of the system in different scenarios.

The simulator is implemented under Matlab 7.1. The Matlab functions that implement functional blocks from fig. 3 are briefly described in the following. Source: generates data blocks (bits of " 1 " and " 0 " respectively) to be jointly encoded by the encoder. Since the later is based on CTC, which uses an incorporated interleaver, the output of the block must be adapted to the size of the subsequent interleaver. This is done via an input parameter of the block.

Encoding: It is known that parallel concatenation of r-input, binary RSC codes offers several advantages in comparison with classical binary turbo codes (TCs). As advantages, we may mention: better convergence of the iterative process and robustness of the decoder[1]. In this work we used duo-binary CTC with rate equal to $1 / 2$. It consists of two convolutional codes, in parallel concatenation, with memory 3 and rate $2 / 3$ and encoder polynomials: 15 (feedback) and 13 (Redundancy) in octal, and one $\mathrm{S}$-interleaver. The S-interleaver is a random type interleaver.

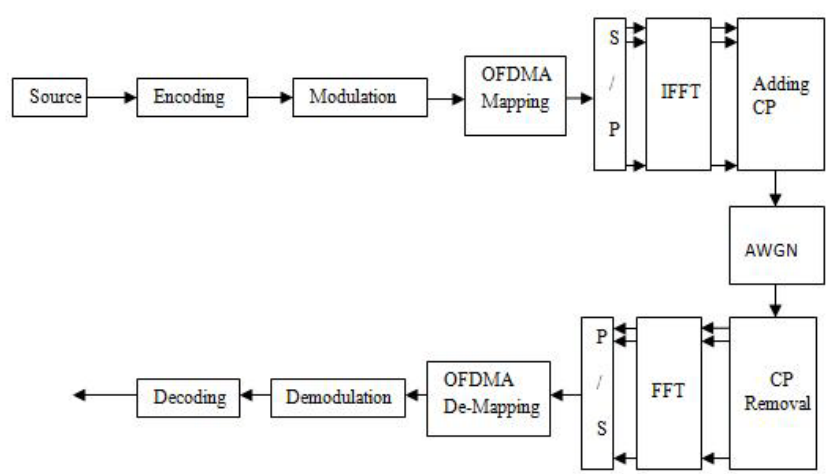

Figure 3. The block scheme of the WiMAX simulator

Therefore, unlike the pure random interleaver, a minimum interleaving distance equal with $\mathrm{S}$ is forced by design. In this case $\mathrm{S}$ has the value 27 .

Modulation: It refers to the base-band modulation that consists on the mapping of the input bits to complex symbols, according to the considered constellation. Via an input parameter, all the digital modulations proposed by the standard (QPSK, 16QAM, 64 QAM) may be simulated. The output of 
this block consists of complex valued modulation symbols.

OFDMA Mapping: This is a complex function, depending on a large number of input parameters. The block implements the permutation strategy and covers the distributed permutation types (DL and UL PUSC and DL FUSC). Among its input parameters, we mention: the total number of carriers in the system, permutation type or permutation base (a critical input parameter for the permutation process that decides how the physical sub-carriers are mapped to subchannels). Another input parameter is the total granted resource that is the number of sub-carriers that will be modulated by data, for the current OFDM symbol. In practice, this resource varies on a frame by frame basis, according to the traffic needs in the cell for a certain moment of time. The output of this block is a sequence of logical indexes that gives a complete map of the sub-carriers: sub-carriers that will be modulated by data, pilot sub-carriers, guard sub-carriers and the DC sub-carrier. This is valuable information for the subsequent block that implements the OFDM modulation.

IFFT: This block implements the OFDM modulation. Under certain conditions, this equation matches relation (1), which allows a simple implementation of the multi-carrier modulator. Only those positions k corresponding to the data sub-carriers (as given by OFDMA mapping) will be modulated by the data. Theoretically, this block is preceded by a serial to parallel converter, and, at its output, another block converts the data back to its serial format. The two converters are practically incorporated by the IFFT transform, as it is implemented in Matlab. Hence, there is no need for explicit implementation of these blocks in our simulator.

Adding CP: The cyclic prefix (CP) consists in a copy of the last samples composing the OFDM symbol in front of it. This function is built according to IEEE $802.16 \mathrm{e}$ specifications, which define 4 possible values for the ratio between the duration of the cyclic prefix and the duration of the useful OFDM symbol, i.e. $\mathrm{G} \in\{14,18,116,132\}$.

Channel: The channel adds a white noise $\mathrm{n}$ of a certain variance to a flat faded variant of the useful signal $\mathrm{x}$, $y=$ ray $x+n$, where ray represents a Rayleigh random variable. We selected the Rayleigh model for the channel to simulate an NLOS communication. The Rayleigh fading channel is a useful model of real-world phenomena in wireless communications[5]. These phenomena include multipath scattering effects, time dispersion, and Doppler shifts that arise from relative motion between the transmitter and receiver. Frequency selective Rayleigh fading is envisioned for further versions of the simulator. An AWGN channel is implemented as well.

Receiver: The complementary blocks are implemented in the receiver: the $\mathrm{CP}$ is removed, sub-carriers are demodulated via the FFT transform, and then sub-carrier de-mapping is performed. The decoding scheme takes "soft inputs" (i.e. I and Q components affected by noise) at its input, whereas at its output we will have directly a "hard decision" on the transmitted bits. The decoder uses the Max-Log-MAP algorithm[2]. This suboptimal version is preferred in practice to optimal MAP due to its low computational complexity while keeping near-optimal performance. We have considered, at the decoder, a maximum iteration number equal to 15 , with stop criterion iteration, based on A Posteriori Probability, APP[3]. BER and BLER statistics may be computed. The present paper represents a continuation of [4] where only the case of AWGN channel was considered.

\section{Simulation Results}

First of all we generated the Rayleigh random variable as the square of the sum of squares of two zero mean Gaussians with the standard deviation equal with $1 / \sqrt{2}$. The corresponding Probability Density Function (PDF) is shown in Figure. 4 . The good quality of the Rayleigh random variable generation algorithm already described can be observed analyzing this figure. Indeed, there is a good superposition of the histogram on the theoretical PDF. The quality of the Rayleigh random variable generation can be improved by increasing the length of the two Gaussians used for this purpose[6].

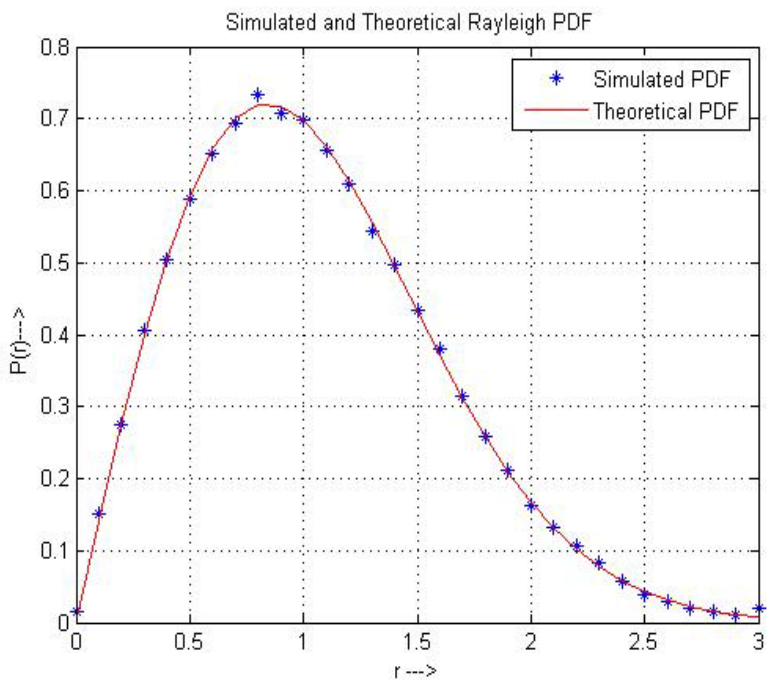

Figure 4. Simulated and theoretical Rayleigh PDF

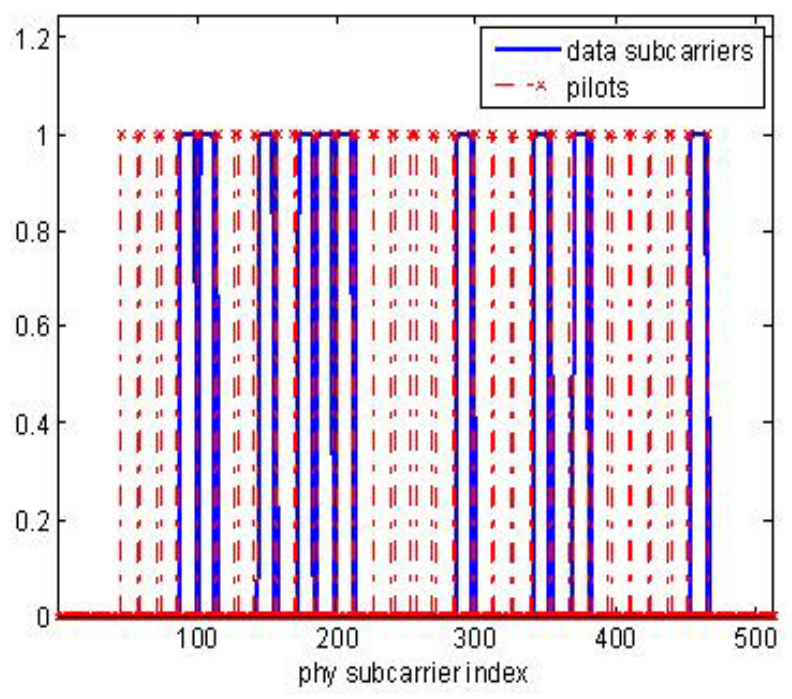

Figure 5. Simulated and theoretical Rayleigh PDF 
Thus by running the simulator, the composition of sub-channels and segments can be identified. Furthermore, the data and pilot carriers may be differentiated. Such a result is shown in fig. 5. In this figure, the sub-carriers that compose segment 0 in DLPUSC 512 are identified in the physical spectrum. In this case, the segment is composed of 120 data sub-carriers, partitioned onto 5 sub-channels. The pilots from the whole spectrum (not only those belonging to segment 0 ) are also shown. We may highlight the concept of frequency diversity: the data sub-carriers belonging to segment 0 are spread over the dedicated bandwidth.

Given the simple channel model (only the white noise perturbing the signal), the permutation type does not make any difference with respect to the BER performance of the system. This would only mater in frequency selective and time variant channels. The following results were obtained for an AWGN and a flat fading Rayleigh channel without mobility using the random variable from Fig. 3 for QPSK and 16QAM modulations, PUSC-DL, PUSC-UL and FUSC-DL permutations.

\section{In AWGN channel:}

The simulation chain was validated by transmitting data through an ideal channel (the block AWGN from the simulator was by-passed) and by measuring the BER. As expected, the BER in this case is 0 , showing that the chain: base-band modulation - encoding - mapping - OFDM modulation works correctly. Next, simulations were carried out in noisy channel conditions. Some results are shown in figures 6, 7 and 8 , for two different permutation types. As expected, the BER performance of the QPSK modulation is better than for the 16QAM case in UL-PUSC 512 (figure. 6).

The results in figure 6 can be improved by replacing the multi-carrier modulation used in WiMAX with single carrier modulation as in LTE[7]. The strength of the turbo-codes leads to very good BER results. Consequently, at $2 \mathrm{~dB}$, BER is already below 0.0001 .

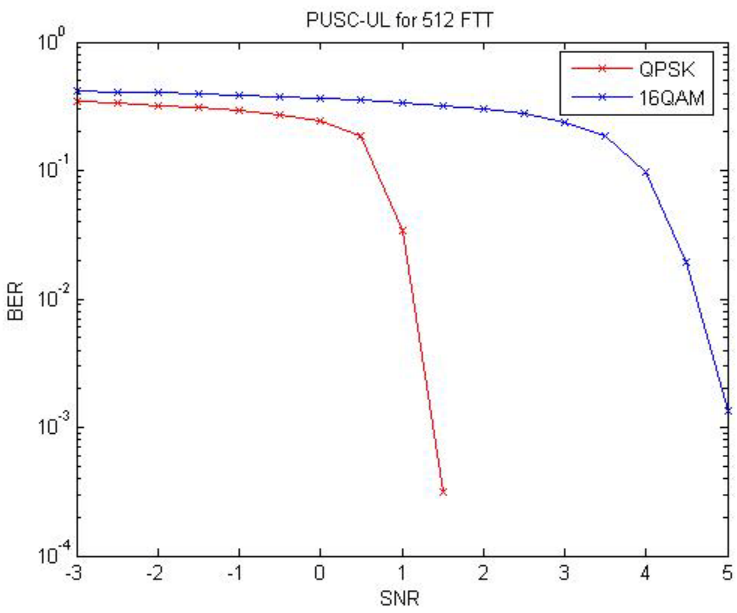

Figure 6. BER performance for UL-PUSC 512

For BER $=0.001$, the gain of QPSK versus 16QAM is approximately $3.5 \mathrm{~dB}$.

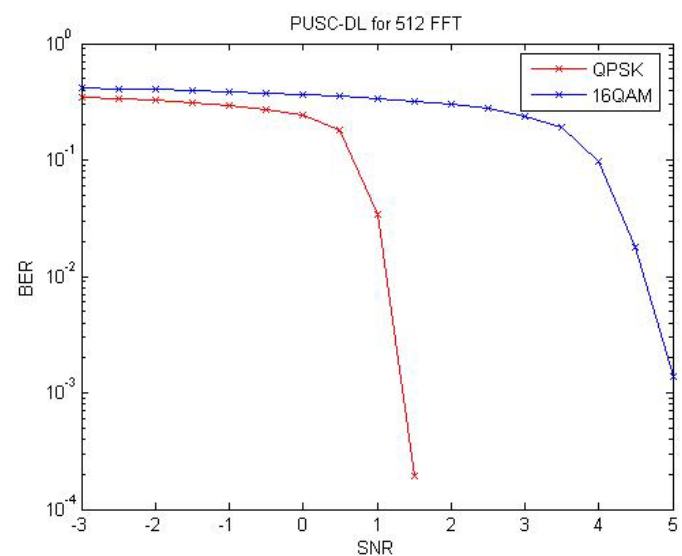

Figure 7. BER performance for DL-PUSC 512

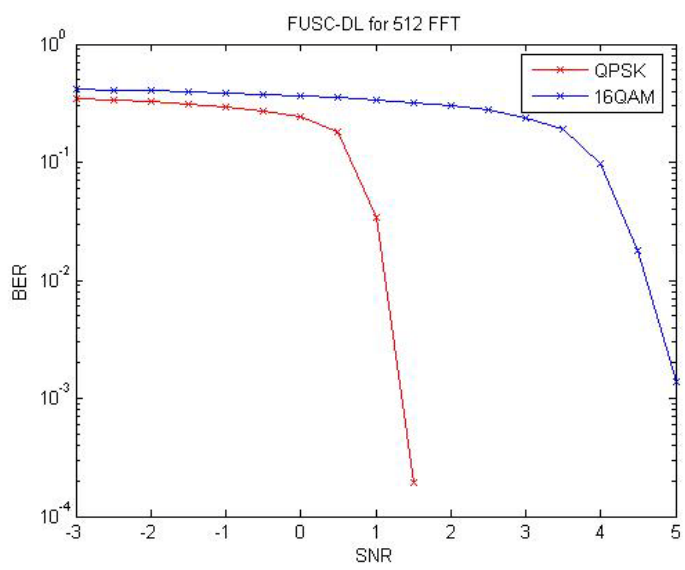

Figure 8. BER performance for DL-FUSC 512

Practically, the same conclusions may be drawn from figures 7 and 8 . This time, the simulation is made for the PUSC-DL and FUSC-DL modes.

In Rayleigh channel:

We simulate the same system but this time with a Rayleigh flat fading channel, and we used the same input parameters in order to compare the simulation results. The results obtained are presented in figures 9-11.

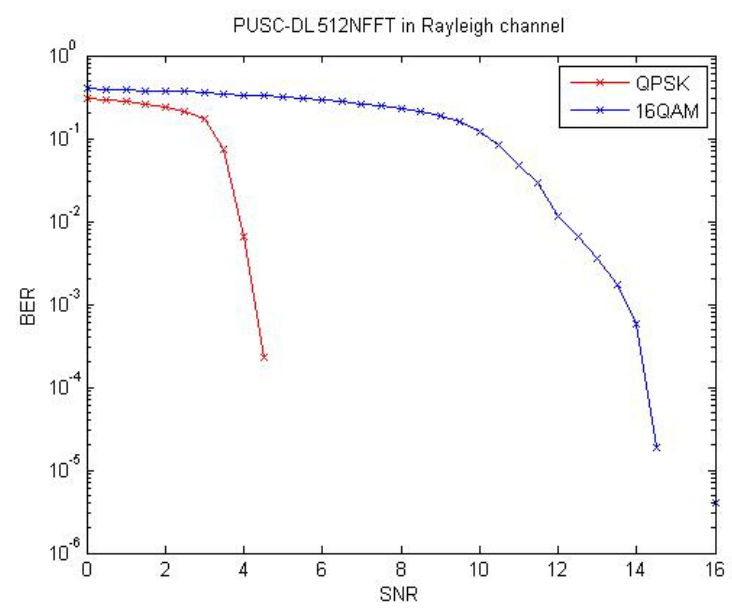

Figure 9. BER performance for DL-PUSC with 512 NFFT 


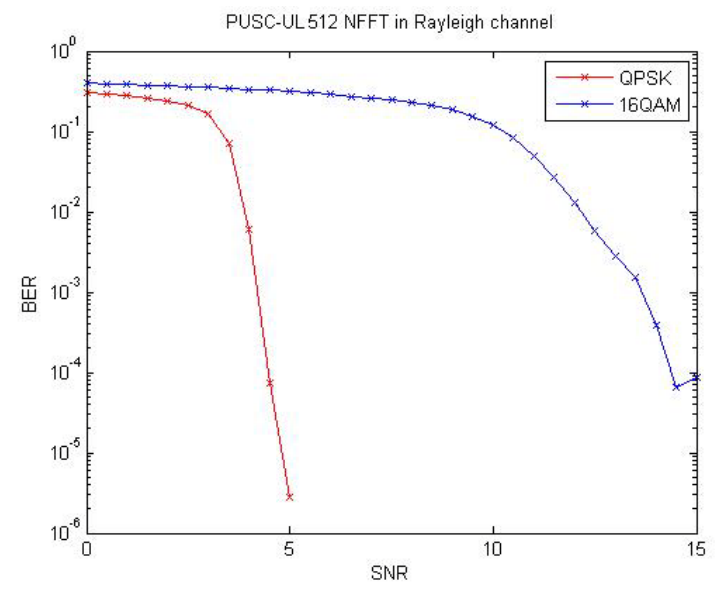

Figure 10. BER performance for UL-PUSC with 512 NFFT

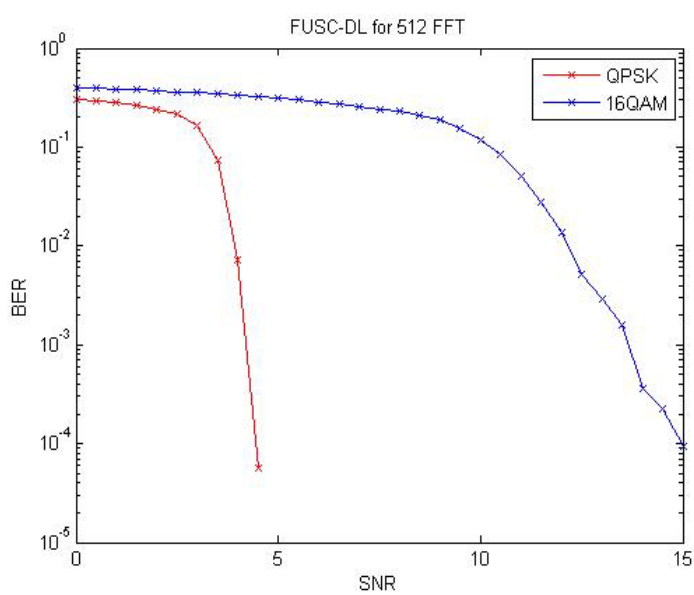

Figure 11. BER performance for DL-FUSC for 512 NFFT

The BER performance of QPSK is better than for 16QAM as it is expected. The strength of the turbo-codes leads to good BER results. Consequently, at $4 \mathrm{~dB}$, BER is below 0.001 . For $B E R=0.001$, the gain of QPSK versus 16 QAM is approximately $9 \mathrm{~dB}$. The BER performance on flat Rayleigh fading channel is poorer than the BER performance on AWGN.

Comparing the experimental results reported in[4] with those presented in figure 9 it can be observed that the fading channel produces a deterioration of performances of $2.5 \mathrm{~dB}$ at $\mathrm{BER}=0.01$ for the QPSK modulation and of $7.5 \mathrm{~dB}$ at the same value of BER for the 16 QAM modulation. This is an important conclusion because it permits the selection of the distance between the mobile station (MS) and the BS where the QPSK can be substituted by the 16 QAM, in the AMC operation mode.

\section{Conclusion and Further Work}

In this paper, we proposed a physical layer simulator for WiMAX in flat Rayleigh fading channel. The simulator allows a better understanding of the signal processing steps taking place at the PHY layer corresponding to the IEEE 802.16e specifications. Furthermore, it allows performance evaluation in different scenarios, through BER and BLER computation. For example, we have highlighted in previous section the deterioration produced by the flat fading and the absence of the line of sight.

The first improvement of the simulator that we consider necessary in the future refers to the channel's model. We only considered the cases of AWGN and flat fading Rayleigh channel without mobility. However, a realistic model for the radio channel must take into account its variability in time (mobility) and its frequency selectivity (multiple paths)[8].

The second improvement will cover some "gaps" in our simulator. Thus, the decoder for 64QAM is not yet implemented. We will extend the implemented permutation types to the adjacent case too, since presently only the distributed permutations are simulated. Finally, we will consider in the future the case of multi-antenna systems[9].

A third category of improvements refers to the programming environment. We have preferred the Matlab because it is adequate for the simulation of different signal processing methods. Its drawback is the low speed of simulation. We intent to translate in the future the functions already implemented in Matlab in C, to increase the simulation speed[7].

\section{ACKNOWLEDGEMENTS}

The research reported in this paper was developed in the framework of a grant funded by the Romanian Research Council (CNCSIS) with the title "Using wavelets theory for decision making" no. 349/13.01.09.

\section{REFERENCES}

[1] C. Douillard and C. Berrou, "Turbo Codes with Rate- $\mathrm{m} /(\mathrm{m}+1)$ Constituent Convolutional Codes", IEEE Transactions on Communications, vol. 53, no. 10, Oct. 2005, pp. 1630-1638

[2] J. Vogt and A. Finger, "Improving the max-log-MAP turbo decoder", Electron. Lett., vol. 36, no. 23, pp. 1937-1939, Nov. 2000

[3] H. Balta, C. Douillard and M. Kovaci, "The Minimum Likelihood APP Based Early Stopping Criterion for Multi-Binary Turbo Codes", Proc. of Symposium of Electronics and Telecommunications-Etc 2006, Timisoara, Sept. 2006, pp. 199-203

[4] M. Oltean, M. Kovaci, J. Mountassir, A. Isar and P. Lazar, "A physical layer simulator for WiMAX", Proc. of the 9th IEEE International Symposium of Electronics and Telecommunications, ISETC 2010, Timisoara, Romania, Nov. 2010, pp. 133-136

[5] Krishna Sankar, "Rayleigh multipath channel model," DSP DSP log, Signal processing for Communication, http://www.dsplog.com/2008/07/14/rayleigh-multipath-chan $\mathrm{nel} /$, posted on July 14, 2008

[6] J. Mountassir, H. Balta, M. Oltean, M. Kovaci, A. Isar, "A Physical Layer Simulator for WiMAX in Rayleigh Fading 
Channel", $6^{\text {th }}$ IEEE International Symposium on Applied Computational Intelligence and Informatics, SACI 2011, Timisoara, Romania, May. 2011pp.281-284

[7] J. C. Ikuno, M. Wrulich, M. Rupp, "System level simulation of LTE networks", 2010 IEEE First Vehicular Technology Conference: VTCS2010, Taipei, Taiwan, 16-19 May, 2010
[8] Mobile WiMAX, edited by Kwang-Cheng Chen and J. Roberto B. de Marca, John Wiley and Sons Ltd, 2008

[9] Han-Kui Chang, Meng-Lin Ku, Li-Wen Huang and Jia-Chin Lin, Multiple Antenna Techniques, in Recent Advances in Wireless Communications, edited by and Jia-Chin Lin, InTech, 2011 